\title{
Outcomes of octogenarians discharged from the hospital after prolonged intensive care unit length of stay after cardiac surgery
}

\author{
Rakesh C. Arora, MD, PhD, FRCSC, ${ }^{a, b}$ Rizwan A. Manji, MD, PhD, MBA, FRCSC, ,a, \\ Rohit K. Singal, MD, MSc, FRCSC, ${ }^{a, b}$ Brett Hiebert, MSc, ${ }^{b}$ and Alan H. Menkis, DDS, MD, FRCSC ${ }^{a, b}$
}

\begin{abstract}
Objective: Octogenarians offered complex cardiac surgery frequently experience a prolonged intensive care unit length of stay; however, minimal data exist on the outcomes of these patients. We sought to determine the rates and predictors of 1-year noninstitutionalized survival ("functional survival") and rehospitalization for octogenarian patients with prolonged intensive care unit length of stay after cardiac surgery and who were discharged from hospital.
\end{abstract}

Methods: The outcomes of discharged patients aged 80 years or more who underwent cardiac surgery with prolonged intensive care unit length of stay ( $>5$ consecutive days) from January 1, 2000, to December 31, 2011, were examined retrospectively from linked clinical and administrative provincial databases. Regression analysis was used to determine predictors of 1-year functional survival and rehospitalization after discharge from the hospital.

Results: A total of 80 of $683(11.7 \%)$ discharged octogenarian patients had prolonged intensive care unit length of stay. Functional survival at 1 year was $92 \%$ and $81 \%$ for those with nonprolonged and prolonged intensive care unit lengths of stay, respectively $(P<.01)$. Lack of outpatient physician visits within 30 days of discharge (hazard ratio, $5.18 ; P<.01$ ) was a significant predictor of poor 1 -year functional survival. The 1 -year rehospitalization rates were $38 \%$ and $48 \%$ for those with nonprolonged and prolonged intensive care unit lengths of stay, respectively, with $41 \%$ of all rehospitalizations occurring within 30 days of initial discharge. A rural residence (hazard ratio, $1.82 ; P<.01$ ) and nosocomial pneumonia during patients' operative admissions (hazard ratio, $2.74 ; P<.01$ ) were associated with rehospitalization within 30 days of discharge.

Conclusions: Octogenarians with prolonged intensive care unit length of stay have acceptable functional survival at 1 year but have high rates of early rehospitalization. Access to health services may influence functional survival and early rehospitalizations. These data suggest that close follow-up of these vulnerable patients after hospital discharge is warranted. ( $\mathrm{J}$ Thorac Cardiovasc Surg 2017;154:1668-78)

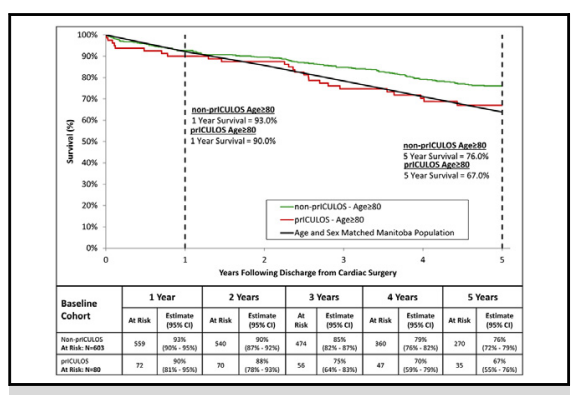

Octogenarians with prolonged ICU LOS have lower functional survival and high rehospitalization rates.

\section{Central Message}

Octogenarians with prolonged ICU LOS have lower functional survival and higher rates of rehospitalization versus those without prolonged ICU LOS. Access to health services may influence outcomes.

\section{Perspective}

With the increasing number of vulnerable older adult patients being referred for cardiac surgery, there is a need to ensure there is effective in-hospital care (eg, preventing nosocomial pneumonia) and adequate community support (eg, adequate access to outpatient physician services within 30 days) to improve postdischarge outcomes.

See Editorial Commentary page 1679.
Increasing Canadian life span during the past 5 decades has resulted in a higher number of people living past 80 years of age. ${ }^{1,2}$ A growing burden of heart disease in an aging population has resulted in cardiac surgery being offered to older and increasingly frail patients with multiple comorbidities. $^{3,4}$ After cardiac surgery, although the
From the ${ }^{\mathrm{a}}$ Department of Surgery, and ${ }^{\mathrm{b}}$ Cardiac Sciences Program, University of Manitoba, Winnipeg, Manitoba, Canada

R.C.A. and R.A.M. have contributed equally as co-first authors for this article. Read at the 96th Annual Meeting of The American Association for Thoracic Surgery, Baltimore, Maryland, May 14-18, 2016.

Received for publication June 1, 2016; revisions received April 13, 2017; accepted for publication April 26, 2017; available ahead of print July 6, 2017.

\footnotetext{
Address for reprints: Rakesh C. Arora, MD, PhD, FRCSC, Department of Surgery, Anesthesia \& Perioperative Medicine and Physiology \& Pathophysiology, University of Manitoba, St Boniface Hospital, CR3012-369 Tache Ave, Winnipeg, Manitoba, Canada R2H 2A6 (E-mail: rakeshcarora@gmail.com). 0022-5223/\$36.00

Copyright () 2017 by The American Association for Thoracic Surgery http://dx.doi.org/10.1016/j.jtcvs.2017.04.083
} 


\section{Abbreviations and Acronyms}

ICU $=$ intensive care unit

LOS $=$ length of stay

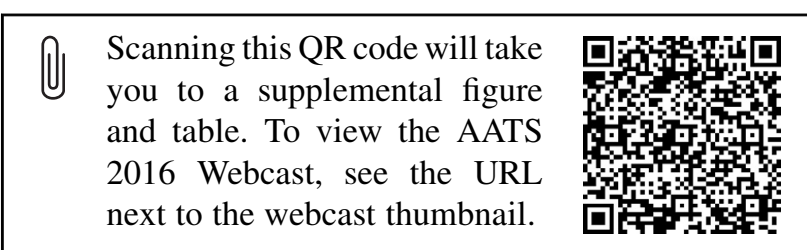

majority of patients require recovery in a postoperative intensive care unit (ICU) environment for less than 48 hours, many patients experience a more complicated course, resulting in a prolonged ICU length of stay (LOS). ${ }^{2-8}$ Patients who have a prolonged ICU LOS after cardiac surgery experience higher rates of rehospitalization with associated worse functional capacity, ${ }^{5}$ worse health-related quality of life, ${ }^{6,7}$ increased healthcare costs, and lower rates of noninstitutionalized survival (ie, being alive and in their own home). ${ }^{8}$

A recent examination of the outcomes of approximately 9500 cardiac surgery cases performed in Manitoba over a 10 -year period has demonstrated that the number of patients who experienced a prolonged ICU LOS after cardiac surgery (defined as requiring an ICU admission for $\geq 5$ days) has increased from 7\% between 2000 and 2009 to greater than $11 \%$ between 2010 and 2013 (a relative increase of $57 \%){ }^{8}$ Furthermore, a significant increase in the numbers of patients aged 80 years or older has been observed at the University of Manitoba (Figure 1). At present, people aged 80 years or more represent the Canadian demographic with the fastest growth rate, more than tripling in number over the last 3 decades. $^{2}$ Therefore, the number of older adult patients undergoing heart surgery and experiencing prolonged ICU LOS will only continue to increase.

Although recent investigations have sought to understand outcomes in octogenarian patients undergoing cardiac surgery, ${ }^{9-12}$ the long-term functional impact for those patients who require prolonged ICU LOS after cardiac surgery remains unclear. This leads to challenges in decision making on the indication and timing of surgery, in conversations with the patient and their caregivers in times of unanticipated outcomes, and in ensuring adequate discharge planning for these vulnerable patients. Therefore, we sought to examine the long-term impact of prolonged ICU LOS on the octogenarian patient to determine predictors for adverse outcomes, such as poor functional survival (ie, dead or institutionalized $)^{8}$ and rehospitalization. We hypothesized that octogenarians with a prolonged ICU LOS have a lower rate of functional survival and higher rates of rehospitalization 1 year after their cardiac surgery procedure.

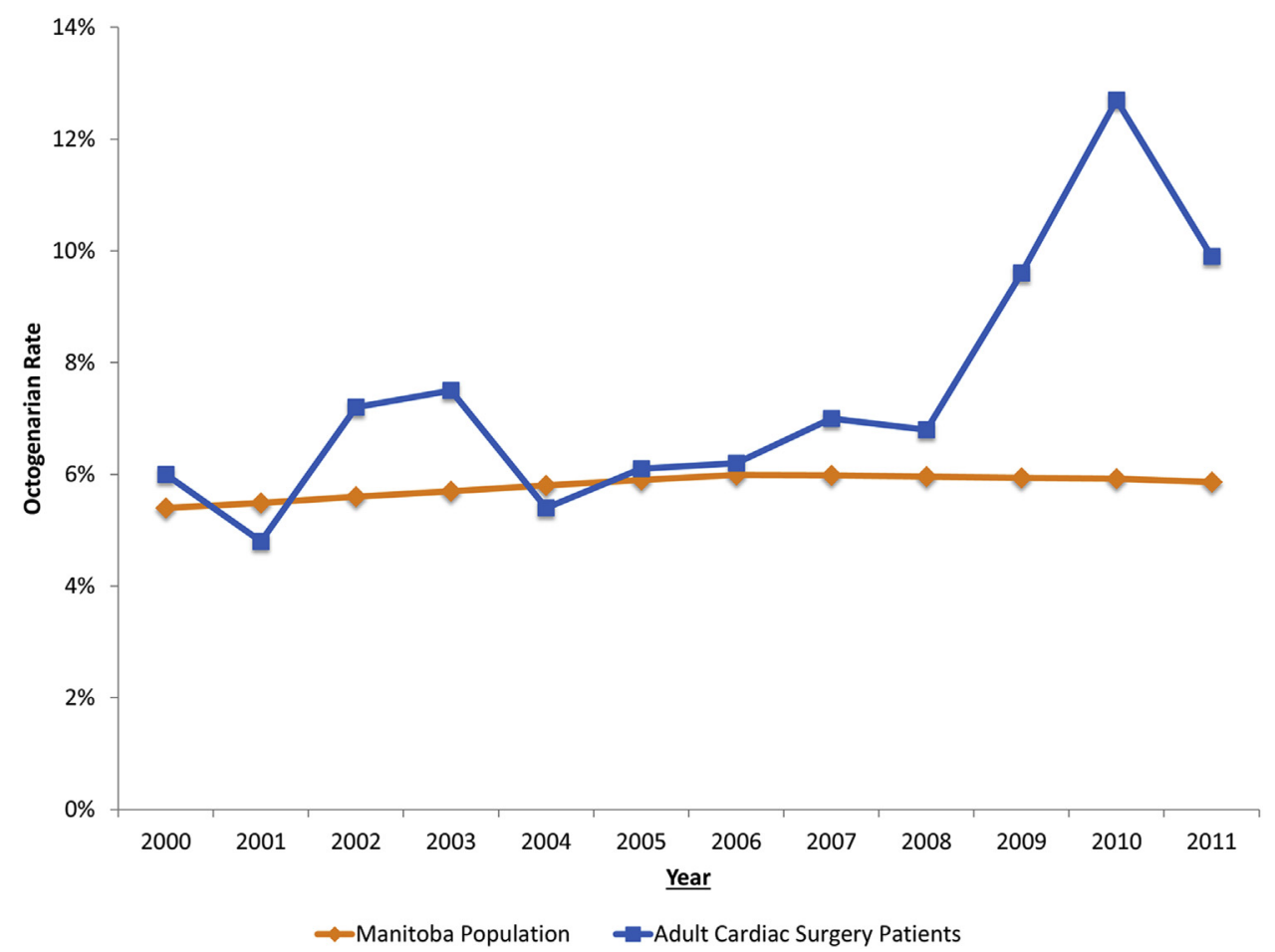

FIGURE 1. Proportion of the Manitoban general and cardiac surgical population aged 80 years or more. The rate of provincial rate of octogenarians (orange line) has been stable, whereas the number of patients undergoing cardiac surgery (blue line) has approximately doubled over a 10-year period. 


\section{METHODS AND MATERIALS}

This was a single-region, single-center retrospective database study. Approval was obtained from the institutional research ethics boards and the Manitoba Health Information Privacy Committee. Informed consent was waived for this retrospective, deidentified database study.

\section{Databases}

The Manitoba Centre for Health Policy at the University of Manitoba's Rady Faculty of Health Sciences in Winnipeg, Manitoba, Canada, houses several provincial clinical and administrative databases within the Population Health Research Data Repository. The clinical data for this study were derived from the Winnipeg Regional Health Authority ICU database and the Manitoba Cardiac Surgical Database as previously described. ${ }^{8}$ Separation abstracts data provided information about an individual's initial cardiac surgery hospitalization and any subsequent rehospitalization. Medical Claims (physician billings) data determined the degree of outpatient interaction a patient had with his/her physician after discharge. Long-term care and vital statistics data provided an individual's functional status (alive and noninstitutionalized). Only new institutional (ie, nursing home or other long-term care facility) admission is included within this definition. If a patient was transferred to another facility before being discharged to home, time to the first rehospitalization or institutionalization was determined from that facility. Social Assistance data provided information on individuals requiring any income assistance before or after cardiac surgery. Costs for the initial cardiac surgery hospitalization and subsequent rehospitalizations occurring within 1 year after hospital discharge were determined by a validated method established by the Manitoba Centre for Health Policy. ${ }^{13}$ This method used the Canadian Institute for Health Information resource intensity weights to measure the intensity of resource use associated with a particular inpatient hospitalization relative to a standard hospitalization. Multiplying the resource intensity weight value by the cost per weighted case (obtained from the 2013/2014 fiscal year) allows one to obtain an approximation of costs associated with each hospitalization in 2013/2014 Canadian dollars.

Publicly available data from Statistics Canada provided neighborhoodlevel income data across the entire province of Manitoba. Each neighborhood was assigned to a provincial income quintile. The databases have been validated and used in a previous study. ${ }^{8}$

\section{Patient Population}

The study population included all patients aged 80 years or more who underwent cardiac surgery between January 1, 2000, and December 31, 2011, from a single healthcare region with a catchment area of approximately 1 million people who were eventually discharged from the hospital. Patients requiring extracorporeal membrane oxygenation for postcardiotomy indications were included. The prolonged ICU LOS cohort was defined as patients requiring 5 or more consecutive days in the ICU after their index cardiac surgery based on an optimal cutpoint determination from our dataset. ${ }^{14}$ Those with prolonged ICU LOS were compared with those without prolonged ICU LOS. Only patients who reside in Manitoba are included in this analysis. Patients who reside outside of Manitoba were not included because it is not possible to link their data with the Provincial data repository.

The primary outcome of interest was "functional survival," defined as alive and not requiring a long-term institutional admission (ie, living in their own home) within 1 year after discharge from their operative hospital admission. ${ }^{8}$ The secondary outcome of interest was early (30-day) and 1-year rehospitalization after discharge to home.

\section{Data Analysis}

Continuous variables were compared using a $t$ test (for parametric data) or Mann-Whitney test (for nonparametric data), and categoric variables were compared using a chi-square or Fisher exact test where appropriate.
Rates of functional survival were calculated using Kaplan-Meier survival curves and compared between those with nonprolonged ICU LOS and those with prolonged ICU LOS using a log-rank test. Rates of first hospital readmission were calculated using cumulative incidence curves with a competing risk of death before rehospitalization for up to 1 year postdischarge, and the values were compared between nonprolonged ICU LOS and prolonged ICU LOS cases using Gray's test.

In addition, a multivariable Cox proportional hazard model was developed to further characterize the factors associated with time to functional survival up to 1 year and first readmission to hospital within 30 days of hospital discharge for the entire study cohort of patients aged 80 years or more. All variables presented in Table 1 taken from both clinical and administrative data sources were considered for the final model. Multiple imputation with a Markov Chain Monte Carlo equation was applied to handle all missing covariate values. A stepwise selection method was used that considered the score test criteria with a $P$ value less than .05 for entry of variables into the model and the Wald test criteria with a $P$ value greater than .05 for removal of selected variables. Statistical analysis was undertaken using SAS software, version 9.3 of the SAS System for Windows (SAS Institute Inc, Cary, NC).

\section{RESULTS}

\section{Patient Demographics}

A total of 743 patients aged 80 years or more underwent a cardiac surgery procedure during the study period (Figure E1). Octogenarians with prolonged ICU LOS experienced a higher in-hospital mortality (primarily in the ICU) rate $(25 / 105=23.8 \%)$ versus the nonprolonged ICU LOS cohort $(35 / 638=5.5 \% ; P<.01)$.

Of 683 patients who underwent cardiac surgery who survived to hospital discharge, $80(11.7 \%)$ experienced a prolonged ICU LOS after their procedure. Octogenarian patients who required prolonged ICU LOS were more commonly female and typically presented with a higher level of acuity, frequently with cardiogenic shock (Table 1).

\section{Operative Characteristics and Intensive Care Unit Complications}

Octogenarian patients with prolonged ICU LOS tended to have undergone a complex procedure (ie, procedure other than isolated coronary artery bypass grafting or valve procedure) and more frequently with higher urgency status and cardiogenic shock (Table 2). The ICU LOS was 5 times longer and total hospital LOS was twice as long in octogenarians with prolonged ICU LOS versus those with nonprolonged ICU LOS (Table 2).

\section{Survival and Functional Survival}

The 1- and 5-year unadjusted survivals of patients aged 80 years or more who underwent cardiac surgery and had prolonged ICU LOS and nonprolonged ICU LOS are shown in Figure 2 with further comparison with the survival curve for an age- and sex-matched provincial population via publically available data from Statistics Canada. Patients aged 80 years or more who did not have prolonged ICU LOS had higher survival after cardiac surgery compared with the age- and sex-matched general population. Figure 3 
TABLE 1. Patient characteristics for octogenarian patients who underwent cardiac surgery and survived to hospital discharge

\begin{tabular}{lcc}
\hline \multicolumn{1}{c}{ Variable } & $\begin{array}{c}\text { Nonprolonged ICU LOS cohort } \\
(\mathbf{n}=\mathbf{6 0 3})\end{array}$ & $\begin{array}{c}\text { Prolonged ICU LOS cohort } \\
(\mathbf{N}=\mathbf{8 0})\end{array}$ \\
\hline Preoperative variables & & $\boldsymbol{P}$ value \\
Age, $\mathrm{y}$ & $82(81-84)$ & $82(81-83)$ \\
Female & $225(37.3 \%)$ & $41(51.3 \%)$ \\
BMI $\left(\mathrm{kg} / \mathrm{m}^{2}\right)$ & $26.9(24.5-29.8)$ & $26.9(24.9-31.2)$ \\
Previous MI & $294(48.8 \%)$ & $39(48.8 \%)$ \\
Diabetes mellitus & $127(21.1 \%)$ & $15(18.8 \%)$ \\
Peripheral vascular disease & $135(22.4 \%)$ & $16(20.0 \%)$ \\
History of arrhythmia & $109(18.1 \%)$ & $20(25.0 \%)$ \\
Congestive heart failure & $105(17.4 \%)$ & $21(26.3 \%)$ \\
COPD & $60(10.0 \%)$ & $9(11.3 \%)$ \\
Renal insufficiency (creatinine $>1.8 \mathrm{mg} / \mathrm{dL})$ & $66(11.0 \%)$ & $13(16.3 \%)$ \\
Cerebrovascular disease & $85(14.1 \%)$ & $13(16.3 \%)$ \\
Previous mental health condition & $93(15.4 \%)$ & $13(16.3 \%)$ \\
Cardiogenic shock & $8(1.3 \%)$ & .63 \\
Ejection fraction & & .63 \\
$\geq 50 \%$ & $388(64.3 \%)$ & .14 \\
$35 \%-49 \%$ & $154(25.5 \%)$ & .06 \\
$<35 \%$ & $61(10.1 \%)$ & .72 \\
Nonelective surgery & $31(5.1 \%)$ & .16 \\
\hline
\end{tabular}

Categoric variables compared using chi-square test. Continuous variables are compared using Mann-Whitney test. Categoric variables are expressed as $\mathrm{N}$ (\%). Continuous variables are expressed as median (quartile 1-quartile 3). ICU, Intensive care unit; $L O S$, length of stay; BMI, body mass index; $M I$, myocardial infarction; $C O P D$, chronic obstructive pulmonary disease.

demonstrates the functional survival (alive and noninstitutionalized) of the studied patient population. Octogenarian patients with prolonged ICU LOS had a lower rate of the primary outcome of functional survival 1 year after discharge from the hospital $(81.3 \%$ prolonged ICU LOS vs $91.7 \%$ nonprolonged ICU LOS; $P<.01)$. This poor functional survival trend continued up to 5 years $(57.6 \%$ vs $70.1 \% ; P<.01)$ from hospital discharge. A Cox proportional hazards model was generated on the total octogenarian cohort, which identified 4 factors associated with poor 1-year functional survival (Table 3). Lack of outpatient physician visits within 30 days of discharge was associated with a 5-fold increased risk of poor 1-year functional survival (hazard ratio, 5.18; $P<.01$ ).

\section{Hospital Readmission}

The times to first rehospitalization rates in octogenarian patients who had survived to hospital discharge after cardiac surgery are shown in Figure 4. The need for rehospitalization was high in all patients aged 80 years or more, with $41 \%$ of rehospitalizations occurring within the first 30 days postdischarge; however, this was not statistically different between those with prolonged ICU LOS and those with nonprolonged ICU LOS. For patients rehospitalized within 1 year of discharge, the median interquartile range time to first rehospitalization was 54 (11-179) days in the nonprolonged ICU LOS cohort versus 41 (12-160) days in the prolonged ICU LOS cohort $(P=.63)$. The total cumulative hospital bed days occupied within the first year of discharge were 5327 days for those with nonprolonged ICU LOS (8.8 days/patient) and 1109 days for those with prolonged ICU LOS (13.9 days/patient) $(P=.12)$. The most common broad category reasons for rehospitalization were heart failure, diabetes, and ischemic heart disease. Regression analysis demonstrated living in a rural setting (hazard ratio, 1.82; 95\% confidence interval, $1.25-2.66 ; P<.01)$ and nosocomial pneumonia during the index cardiac surgery hospitalization (hazard ratio, 2.74; $95 \%$ confidence interval, $1.28-5.90 ; P<.01)$ as associated with the time to first rehospitalization within 30 days of discharge in patients aged 80 years undergoing cardiac surgery.

\section{Costs}

The total median (interquartile range) cost within the first year of cardiac surgery for patients aged 80 years or more who were discharged from the hospital was $\$ 33,945(\mathrm{C} \$ 24,798-\mathrm{C} \$ 50,874)$ for those with nonprolonged ICU LOS versus \$75,798 (C\$53,471$\mathrm{C} \$ 127,624)$ for those with prolonged ICU LOS $(P<.01)$. These costs were largely due to initial hospitalization costs (vs rehospitalization costs). The index cardiac surgery hospital costs were $\$ 29,483(\$ 22,036-\$ 39,419)$ for those with nonprolonged ICU LOS and \$65,658 $(\$ 46,010-\$ 103,612)$ $(P<.01)$ for those with prolonged ICU LOS.

\section{DISCUSSION}

In this analysis, we sought to understand the impact of functional survival $^{8}$ and early rehospitalization in a 
TABLE 2. Operative, intensive care unit, and postdischarge patient characteristics for octogenarian patients undergoing cardiac surgery

\begin{tabular}{|c|c|c|c|}
\hline Variable & $\begin{array}{l}\text { Nonprolonged } \\
\text { ICU LOS cohort } \\
(n=603)\end{array}$ & $\begin{array}{c}\text { Prolonged } \\
\text { ICU LOS cohort } \\
(\mathbf{N}=\mathbf{8 0})\end{array}$ & $P$ value \\
\hline \multicolumn{4}{|l|}{ Operative variables } \\
\hline Procedure type & & & $<.01$ \\
\hline Isolated CABG & $362(60.0 \%)$ & $32(40.0 \%)$ & \\
\hline Single non-CABG & $106(17.6 \%)$ & $13(16.3 \%)$ & \\
\hline$\geq 2$ procedures & $135(22.4 \%)$ & $35(43.8 \%)$ & \\
\hline Crossclamp time (min) & $65(48-98)$ & $86(52-126)$ & $<.01$ \\
\hline Cardiopulmonary bypass time (min) & $103(77-141)$ & $127(88-184)$ & $<.01$ \\
\hline \multicolumn{4}{|l|}{ Postoperative variables } \\
\hline APACHE II score & $16(14-18)$ & $19(16-22)$ & $<.01$ \\
\hline APACHE II $\geq 20$ & $92(15.3 \%)$ & $37(46.3 \%)$ & $<.01$ \\
\hline Mechanical ventilation (d) & $1(0-2)$ & $5(2-7)$ & $<.01$ \\
\hline Reintubation & $9(1.5 \%)$ & $17(21.3 \%)$ & $<.01$ \\
\hline \multicolumn{4}{|l|}{ Blood products } \\
\hline Red blood cells (units) & $1(0-2)$ & $3(2-6)$ & $<.01$ \\
\hline Fresh-frozen plasma (units) & $0(0-0)$ & $2(0-6)$ & $<.01$ \\
\hline Platelets (units) & $0(0-0)$ & $0(0-5)$ & $<.01$ \\
\hline \multicolumn{4}{|l|}{ ICU complications } \\
\hline Tachyarrhythmias & $71(11.8 \%)$ & $46(57.5 \%)$ & $<.01$ \\
\hline Other cardiovascular procedure (sternal rewiring, returns to operating room, other) & $42(7.0 \%)$ & $28(35.0 \%)$ & $<.01$ \\
\hline Thrombocytopenia & $44(7.3 \%)$ & $18(22.5 \%)$ & $<.01$ \\
\hline Cardiogenic shock & $30(5.0 \%)$ & $26(32.5 \%)$ & $<.01$ \\
\hline Delirium & $22(3.7 \%)$ & $12(15.0 \%)$ & $<.01$ \\
\hline Congestive heart failure & $15(2.5 \%)$ & $7(8.8 \%)$ & $<.01$ \\
\hline Other pulmonary/thoracic procedures & $7(1.2 \%)$ & $12(15.0 \%)$ & $<.01$ \\
\hline Acute renal insufficiency (creatinine $>1.8 \mathrm{mg} / \mathrm{dL}$ ) & $18(3.0 \%)$ & $16(20.0 \%)$ & $<.01$ \\
\hline \multicolumn{4}{|l|}{ LOS } \\
\hline ICU LOS (d) & $1.2(0.9-2.6)$ & $6.8(5.8-9.9)$ & $<.01$ \\
\hline Hospital LOS (d) & $13(8-21)$ & $30(22-51)$ & $<.01$ \\
\hline Postoperative follow-up with any physician & & & .05 \\
\hline No physician visits within $30 \mathrm{~d}$ of discharge & $56(9.3 \%)$ & $14(17.5 \%)$ & \\
\hline $1-4$ visits within $30 \mathrm{~d}$ of discharge & $478(79.3 \%)$ & $55(68.8 \%)$ & \\
\hline $5+$ visits within $30 \mathrm{~d}$ of discharge & $69(11.4 \%)$ & $11(13.8 \%)$ & \\
\hline
\end{tabular}

Categoric variables compared using chi-square test. Continuous variables compared using Mann-Whitney test. Categoric variables expressed as $\mathrm{N}(\%)$. Continuous variables expressed as median (quartile 1-quartile 3). ICU, Intensive care unit; $L O S$, length of stay; CABG, coronary artery bypass grafting; APACHE, Acute Physiology And Chronic Health Evaluation.

particularly vulnerable group of patients with prolonged ICU LOS, namely, those aged 80 years or more. This study identified that, in general, octogenarian patients with a prolonged ICU LOS tended to have undergone a complex procedure, frequently on an urgent or emergency basis and often in cardiogenic shock. This affected both short-term (1-year) and long-term (5-year) functional survival, although functional survival was still reasonable at more than $80 \%$ for 1 year and more than $50 \%$ at 5 years (Figure 3). In addition, all patients aged 80 years or more experienced high rates of early rehospitalization, although most of the costs for the first year after the surgical procedure were encountered during the index cardiac surgery hospitalization. Although there have been several important reports examining short- and long-term outcomes in patients aged 80 years or more, the strength of this analysis was the inclusion of a large cohort of patients with mixed procedure type and longitudinal outcome (up to 5 years), as well as cost data. With an increasing number of octogenarians presenting for cardiac surgery, understanding the outcomes of these patients and factors that can change the outcomes is increasingly important for patients, families, healthcare providers, and administrators.

With recent important reports examining outcomes in the now aging population undergoing cardiac surgery, hospital discharge is frequently cited as the metric to a "successful" procedural outcome. ${ }^{11}$ However, as demonstrated in this and previous analyses, ${ }^{12,15,16}$ survival after a complicated ICU and hospital stay does not necessarily translate to "thriving" in the community after hospital discharge. In the current era of cardiac surgery, there is a need to further understand patient-centered outcomes. Indeed, 


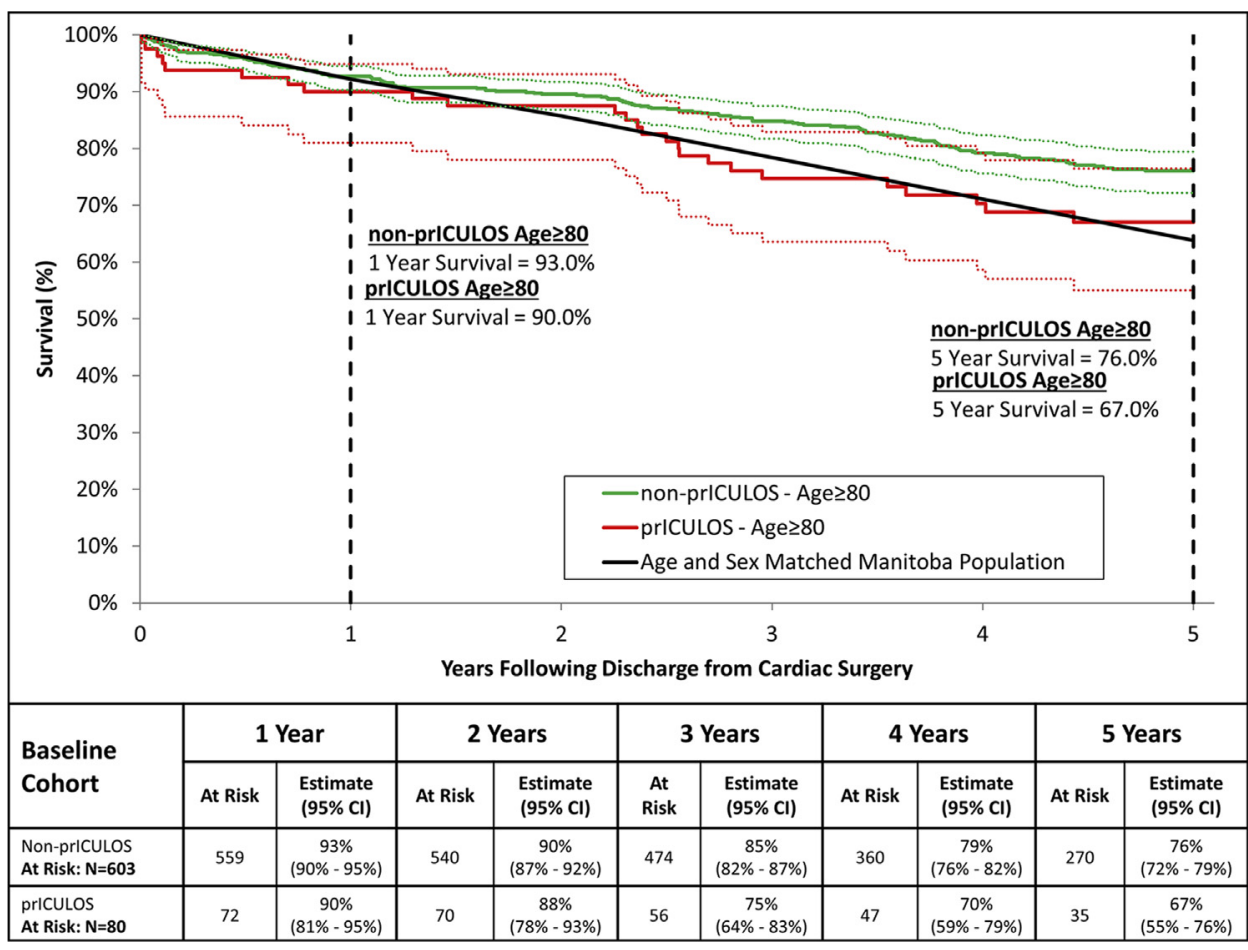

FIGURE 2. Kaplan-Meier survival curve: 5-year survival by ICU LOS in patients aged 80 years or more compared with the age- and sex-matched Manitoba population survival curve ( $95 \%$ confidence intervals indicated by the dashed line). Note that patients with nonprolonged ICU LOS after cardiac surgery have better survival compared with an age- and sex-matched provincial population. $P$ value of .06 (log rank) comparing nonprolonged ICU LOS and prolonged ICU LOS. prICULOS, Prolonged intensive care unit length of stay; $C I$, confidence interval.

functional capacity is increasingly recognized as an important patient-centric value, perhaps more so than hospital survival, to older adult patients undergoing cardiac surgery. ${ }^{5-7,17}$ Bashour and colleagues ${ }^{5}$ have promoted this in their recommendation that understanding outcomes of the patient with prolonged ICU LOS is most appropriately examined in the long-term follow-up of these patients after discharge from their index hospital admission.

The clinical significance of this and other important work examining outcomes in the older adult undergoing cardiac surgery is important to understand current and predict future rates of healthcare resource use. Second, it is anticipated that knowledge of short- and long-term functional outcomes can facilitate the informed consent and shared decision-making processes with the patient and their caregivers. It is increasingly clear that even complex operations can be performed in patients aged more than 80 years, ${ }^{12,18}$ and this is supported by the data in this analysis wherein the 5 -year survival curve of those with nonprolonged ICU LOS cardiac surgery appears higher than in the age-matched general (noncardiac surgery) population, an important verification of the suitability of this treatment for octogenarians. Conversely, octogenarian patients who require a prolonged ICU stay experience lower rates of functional survival after their cardiac surgery procedure. Furthermore, the "successfully" discharged octogenarian patient still appears to experience higher rates of rehospitalization. The lack of access to timely physician visits was associated with a significantly lower rate of functional survival. This is in keeping with recent frailty theories that propose that "damage begets damage," which may, in part, account for the persistent decrease in functional survival 5 years from the initial cardiac surgical procedure. ${ }^{19}$ Therefore, further work to identify processes of care that require optimization predischarge and postdischarge to improve the patient journey and ensure that patients continue to successfully recover in the community after hospital discharge is needed.

It seems that a "multi-armed" approach to improve outcomes for octogenarians undergoing cardiac surgery may be considered on the basis of the data generated from this study. The first relates to preoperative referral. A notable finding from this analysis was the higher proportion of female patients and patients with high urgency in the prolonged ICU LOS group. It is unclear whether this represents a referral bias in which older (and perhaps female) patients are not being referred early in their disease process, are refusing to undergo a surgical procedure at the initial consultation, or are presenting with more advanced disease. Although it is not possible to completely understand this phenomenon, it is worthy to consider that patients, their families, and physicians may be pursuing surgical therapies only as a last resort, leading to late referral. Given the results 


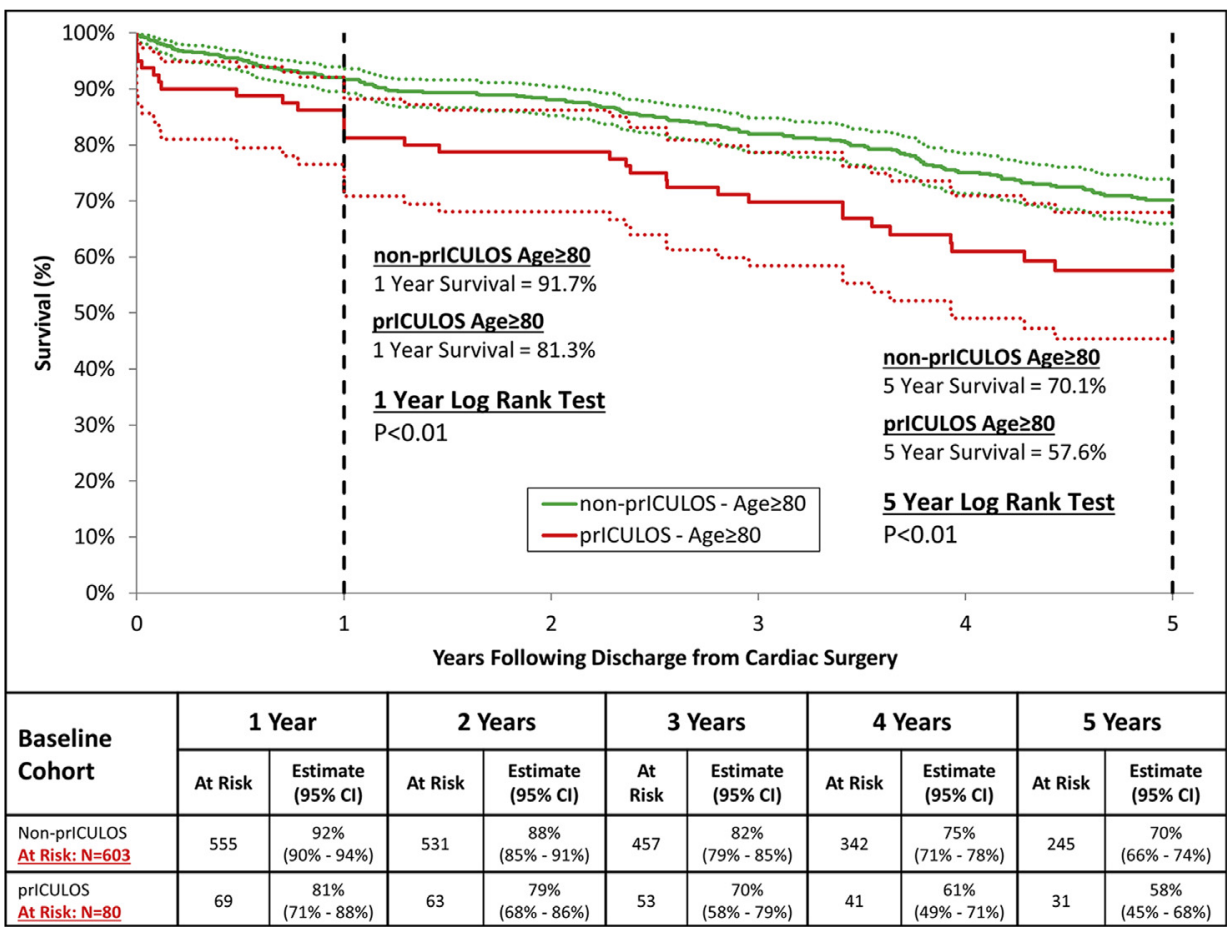

FIGURE 3. Kaplan-Meier survival curve: 5 -year functional survival (alive and not institutionalized) by ICU LOS in patients aged 80 years or more who were discharged from the hospital alive. Functional survival was significantly lower in patients who experienced a prolonged ICU LOS (prolonged ICU LOS, red line) versus those who did not (nonprolonged ICU LOS, green line) after their cardiac surgery procedure at 1 and 5 years (95\% confidence intervals indicated by the dashed line). prICULOS, Prolonged intensive care unit length of stay; $C I$, confidence interval.

of the lower-acuity patients in our study, it is reasonable to diminish apprehension around cardiac surgery for octogenarians and promote early surgical consultation.

The second prong relates to the management of patients experiencing prolonged ICU LOS postoperatively and on transition to the community. In this analysis, important and potentially actionable factors of negative 1-year outcomes for the specific group of patients who have experienced prolonged ICU LOS were identified. For example, quality-improvement initiatives that seek to prevent nosocomial infection may have benefits in this vulnerable population to avoid rehospitalizations. ${ }^{20}$ However, the transition from a tertiary care institution to a community is likely not as readily addressed. Octogenarian patients with prolonged ICU LOS may have to deal with multisystem healthcare issues that are overwhelming for themselves, their caregivers, and their primary care practitioner. ${ }^{21,22}$ At present, there seems to be a lack of a comprehensive approach for these patients, particularly for the rural patient. ${ }^{23}$ With newer minimally invasive procedures now being performed in frail octogenarian and nonagenarian patients previously deemed inoperable, it has become critically important for the healthcare systems to develop strategies to improve clinical outcomes and provide patient and caregiver support. ${ }^{24-28}$ As such, it may be appropriate to consider new models of care using a team-based healthcare delivery model to provide comprehensive medical care to patients with the goal of obtaining maximized health outcomes after hospital discharge. ${ }^{29}$

\section{Study Limitations}

We were limited by the nature of a retrospective design and reporting data from a single tertiary care center. Furthermore, despite examining outcomes over a 10 -year period, a sample size of 80 patients limits the ability of additional subgroup analysis or examination of different LOS cutpoints. In addition, we were limited to the use of aggregate data that prevented more granular insight into the causes of in-hospital mortality in the octogenarian with prolonged ICU LOS. However, we were able to link our surgical database with a detailed provincial administrative data repository to obtain approximately $100 \%$ complete data on patient outcomes in our dataset. Second, although we were able to report on the "traditional" patient factors that inform perioperative risk, we did not capture information that categorizes levels of frailty that have been shown to influence postoperative outcomes. $^{30-33}$ Previous investigations have examined the role of preoperative exercise interventions in patients undergoing cardiac surgery ("prehabilitation"), ${ }^{34,35}$ with a current study under way examining its utility in the frail older adult. ${ }^{36}$ Third, the process of discharge from the ICU to the ward and the ward to home, although undergoing significant evolution over time, still relies on some degree of subjective medical decision making. This creates a 


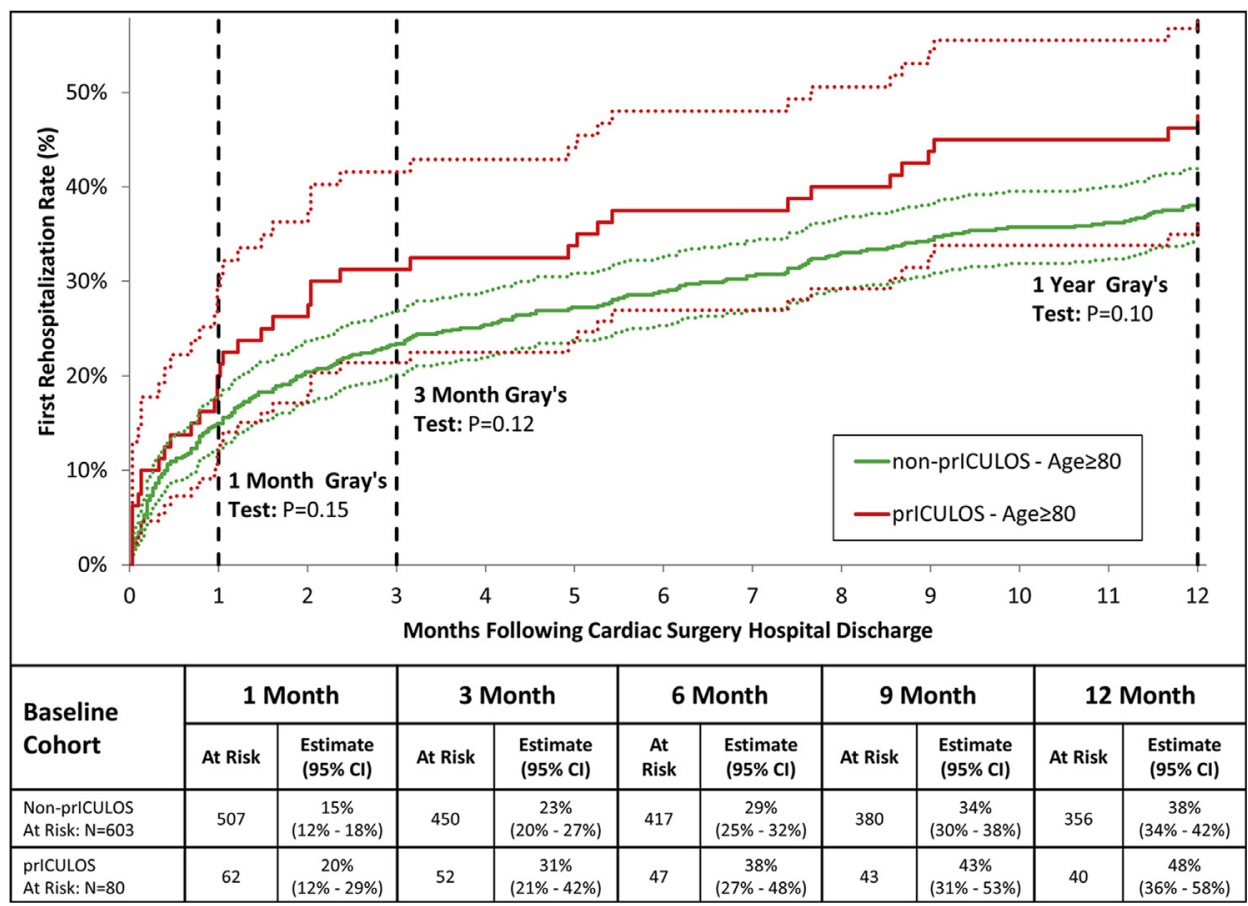

FIGURE 4. Cumulative incidence curve: 1 -year first rehospitalization rates by ICU LOS for patients aged 80 years or more undergoing cardiac surgery. The 1-year rehospitalization cumulative incidence curve for patients aged 80 years or more with (red line) or without (green line) a prolonged ICU LOS (prolonged ICU LOS) after the cardiac surgery procedure demonstrates that rehospitalization rates are high for octogenarians but statistically similar based on ICU LOS (95\% confidence intervals indicated by the dashed line). prICULOS, Prolonged intensive care unit length of stay; $C I$, confidence interval.

possibility that patient discharge is not taking place appropriately on an institutional basis that would have a significant impact on LOS and potential for readmission. There are many facets of our clinical program that attempt to mitigate this possibility. As of 2007, we have a 24-hour in-house intensivist physician staffing model. ${ }^{37,38}$ Decisions to discharge a patient are made on daily bedside rounds with the interdisciplinary team consisting of the medical doctor, physiotherapist, nursing, pharmacy, respiratory therapist, and dietician. Patients who are not ready for transfer typically are reevaluated by 3 PM to determine whether transfer to the ward is appropriate at that stage. For the ward to discharge home (or elsewhere), our team holds 2 interdisciplinary disciplinary meetings per week that involve clinical assistants/physician assistants, nurses, physiotherapy and occupational therapy, social work, and home care service. In general, patients are sent home when all team members are in agreement that they are capable with appropriate support in place. This includes specific physiotherapy criteria, including safe transfers, walking, and climbing stairs. Given the thorough and multidisciplinary nature of this process, we believe that hospital discharges take place appropriately at our institution and potentially even on a somewhat conservative basis lower the likelihood of premature discharge. Fourth, the process of care for the octogenarian after hospital discharge was not fully clear. Although we are able to determine whether a patient was living in his/ her own home (vs a personal care home or another type of long-term care facility), we are unable to determine what level of in-home services may have been required by the patient and caregivers. Last, we did not have detailed information on health-related quality of life, patient and caregiver stress burden, and lost revenue, which certainly have an impact on patient-specific outcomes. Our team is currently examining several of these limitations in a prospective fashion.

\section{CONCLUSIONS}

This retrospective analysis of 683 patients aged 80 years or more who underwent cardiac surgery and survived to

TABLE 3. Multivariable Cox proportional hazards regression variables associated with time to death or institutionalization up to 1 year postdischarge for octogenarians

\begin{tabular}{lccc}
\hline \multicolumn{1}{c}{ Variable } & HR & $\mathbf{9 5 \%}$ CI & $\boldsymbol{P}$ value \\
\hline Diabetes mellitus & 2.86 & $1.68-4.85$ & $<.01$ \\
APACHE II $\geq 20$ & 1.90 & $1.08-3.34$ & .03 \\
Hospital LOS (per day) & 1.02 & $1.01-1.02$ & $<.01$ \\
$\begin{array}{l}\text { No physician visits vs 1-4 physician } \\
\text { visits within 30 d of discharge }\end{array}$ & 5.18 & $2.93-9.16$ & $<.01$ \\
\hline
\end{tabular}

HR, Hazard ratio; $C I$, confidence interval; APACHE, Acute Physiology And Chronic Health Evaluation; $L O S$, length of stay. 
hospital discharge found that those who experienced prolonged ICU LOS have significantly lower, but acceptable functional survival at 1 and 5 years compared with those without prolonged ICU LOS. Furthermore, those with prolonged ICU LOS had a higher proportion of women, high acuity, and cardiogenic shock, suggesting possible referral biases that may need to be addressed, especially considering that patients with nonprolonged ICU LOS had better survival then the age- and sex-matched general population. In addition, all patients aged 80 years or more experienced high rates of rehospitalization after cardiac surgery. The development of healthcare processes that assist the transition of the octogenarian patient who underwent cardiac surgery from a tertiary care center to the community is warranted.

\section{Webcast}

You can watch a Webcast of this AATS meeting presentation by going to: http://webcast.aats.org/2016/Video/ Monday/05-16-16_Room_337_1600_Arora-800.mp4.

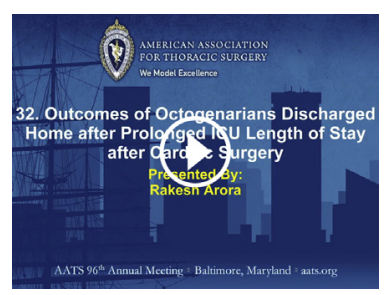

\section{Conflict of Interest Statement}

R.C.A. and R.A.M. have received research grant funding from the Department of Surgery, University of Manitoba, for this work. R.C.A. has received an unrestricted educational grant from Pfizer Canada Inc, for work unrelated to this work. All other authors have nothing to disclose with regard to commercial support.

The authors thank the Manitoba Centre for Health Policy for the use of data contained in the Population Health Research Data Repository under Project \#2013-021 (HIPC \#2013/201408). The results and conclusions are those of the authors, and no official endorsement by the Manitoba Centre for Health Policy, Manitoba Health, or other data providers is intended or should be inferred. Data used in this study are from the Population Health Research Data Repository housed at the Manitoba Centre for Health Policy, University of Manitoba, and were derived from data provided by Manitoba Health, the Winnipeg Regional Health Authority, Vital Statistics Manitoba, and Manitoba Jobs \& The Economy. The authors also thank the Manitoba Vital Statistics Agency.

\section{References}

1. Statistics Canada. Annual Demographic Estimates: Canada, Provinces and Territories. Catalogue No. 91-215-X.; 2014. Available at: http://www.statcan.gc. ca/pub/91-215-x/91-215-x2014000-eng.pdf. Accessed March 23, 2016.
2. Center on Aging, University of Manitoba. Profile of Manitoba's Seniors; 2010. Available at: http://www.gov.mb.ca/shas/publications/docs/profile_manitoba_ senior_2010.pdf. Accessed March 23, 2016.

3. Ferguson TB, Hammill BG, Peterson ED, DeLong ER, Grover FL. A decade of change-risk profiles and outcomes for isolated coronary artery bypass grafting procedures, 1990-1999: a report from the STS National Database Committee and the Duke Clinical Research Institute. Ann Thorac Surg. 2002;73:480-9.

4. Abramov D, Tamariz MG, Fremes SE, Guru V, Borger MA, Christakis GT, et al. Trends in coronary artery bypass surgery results: a recent, 9-year study. Ann Thorac Surg. 2000;70:84-90.

5. Bashour CA, Yared JP, Ryan TA, Rady MY, Mascha E, Leventhal MJ, Starr NJ. Long-term survival and functional capacity in cardiac surgery patients after prolonged intensive care. Crit Care Med. 2000;28:3847-53.

6. Deschka H, Schreier R, El-Ayoubi L, Erler S, Alken A, Wimmer-Greinecker G. Survival, functional capacity, and quality of life after cardiac surgery followed by long-term intensive care stay. J Thorac Cardiovasc Surg. 2013;61:696-700.

7. Bapat V, Allen D, Young C, Roxburgh J, Ibrahim M. Survival and quality of life after cardiac surgery complicated by prolonged intensive care. J Card Surg. 2001;20:212-7.

8. Manji RA, Arora RC, Singal RK, Hiebert B, Moon MC, Freed DH, Menkis AH, et al. Long-term outcome and predictors of noninstitutionalized survival subsequent to prolonged intensive care unit stay after cardiac surgical procedures. Ann Thorac Surg. 2016;101:56-63.

9. Singh AK, Maslow AD, Machan JT, Fingleton JG, Feng WC, Schwartz C, et al. Long-term survival after use of internal thoracic artery in octogenarians is gender related. J Thorac Cardiovasc Surg. 2015;150:891-9.

10. Cappabianca G, Ferrarese S, Musazzi A, Terrieri F, Corazzari C, Matteucci M, Beghi C. Predictive factors of long-term survival in the octogenarian undergoing surgical aortic valve replacement: 12-year single-centre follow-up. Heart Vessels. 2016;31:1798-805.

11. Khounlaboud M, Donal E, Auffret V, Anselmi A, Ingels A, Flécher E, et al. Comparison of preoperative and postoperative characteristics in octogenarians having isolated surgical aortic valve replacement before versus after introduction of transcatheter aortic valve implantation. Am J Cardiol. 2015;116:933-7.

12. Deschka H, Machner M, Welp H, Dell'Aquila AM, Erler S, WimmerGreinecker G. Cardiac reoperations in octogenarians: do they really benefit? Geriatr Gerontol Int. 2016;16:1138-44

13. Altarabsheh SE, Deo SV, Rababa'h AM, Lim JY, Cho YH, Sharma V, et al. Offpump coronary artery bypass reduces early stroke in octogenarians: a metaanalysis of 18,000 patients. Ann Thorac Surg. 2015;99:1568-75.

14. Williams B, Mandrekar J, Mandrekar S, Cha S, Furth A. Finding Optimal Cutpoints for Continuous Covariates with Binary and Time-to-Event Outcomes. Mayo Foundation Technical Report Series. Rochester, MN: Mayo Foundation; 2006.

15. Leung Wai Sang S, Chaturvedi RK, Iqbal S, Lachapelle K, de Varennes B. Functional quality of life following open valve surgery in high-risk octogenarians. $J$ Card Surg. 2012;27:408-14.

16. Aydin S, Yavuz T, Duver H, Kutsal A. Quality of life in the elderly after coronary bypass surgery. Int Heart J. 2006;47:59-65.

17. Konopad E, Noseworthy TW, Johnston R, Shustack A, Grace M. Quality of life measures before and one year after admission to an intensive care unit. Crit Care Med. 1995;23:1653-9.

18. Sorabella RA, Wu YS, Bader A, Kim MJ, Smith CR, Takayama H, et al. Aortic root replacement in octogenarians offers acceptable perioperative and late outcomes. Ann Thorac Surg. 2016;101:967-72.

19. Taneja S, Mitnitski AB, Rockwood K, Rutenberg AD. Dynamical network model for age-related health deficits and mortality. Phys Rev E. 2016;93:22309.

20. Stamou SC, Camp SL, Reames MK, Skipper E, Stiegel RM, Nussbaum M, et al. Continuous quality improvement program and major morbidity after cardiac surgery. Am J Cardiol. 2008;102:772-7.

21. Sveinsdóttir H, Ingadóttir B. Predictors of psychological distress in patients at home following cardiac surgery: an explorative panel study. Eur J Cardiovasc Nurs. 2012;11:339-48.

22. Reay PA, Horner B, Duggan R. The patient's experience of early discharge following total hip replacement. Int J Orthop Trauma Nurs. 2015;19:131-9.

23. Brundisini F, Giacomini M, DeJean D, Vanstone M, Winsor S, Smith A. Chronic disease patients' experiences with accessing health care in rural and remote areas: a systematic review and qualitative meta-synthesis. Ont Health Technol Assess Ser. 2013;13:1-33.

24. Nicolaou G, Ismail M, Cheng D. Thoracic endovascular aortic repair: update on indications and guidelines. Anesthesiol Clin. 2013;31:451-78. 
25. Greenhalgh RM, Brown LC, Powell JT, Thompson SG, Epstein D. Endovascular repair of aortic aneurysm in patients physically ineligible for open repair. $N$ Engl J Med. 2010;362:1872-80.

26. Lederle FA, Freischlag JA, Kyriakides TC, Matsumura JS, Padberg FT Jr, Kohler TR, et al. Long-term comparison of endovascular and open repair of abdominal aortic aneurysm. $N$ Engl J Med. 2012;367:1988-97.

27. Leon MB, Smith CR, Mack M, Miller DC, Moses JW, Svensson LG, et al. Transcatheter aortic-valve implantation for aortic stenosis in patients who cannot undergo surgery. N Engl J Med. 2010;363:1597-607.

28. Deutsch M-A, Krane M, Bleiziffer S, Lange R. Health-related quality of life after transcatheter aortic valve replacement. J Am Coll Cardiol. 2013;61:108.

29. Hickman LD, Phillips JL, Newton PJ, Halcomb EJ, Al Abed N, Davidson PM. Multidisciplinary team interventions to optimise health outcomes for older people in acute care settings: a systematic review. Arch Gerontol Geriatr. 2015;61:322-9.

30. Afilalo J, Mottillo S, Eisenberg MJ, Alexander KP, Noiseux NĆ, Perrault LP, et al. Addition of frailty and disability to cardiac surgery risk scores identifies elderly patients at high risk of mortality or major morbidity. Circ Cardiovasc Qual Outcomes. 2012;5:222-8.

31. Lee DH, Buth KJ, Martin BJ, Yip AM, Hirsch GM. The impact of frailty on outcomes after cardiac surgery: a systematic review. J Thorac Cardiovasc Surg. 2014;148:3110-7.

32. Lee DH, Buth KJ, Martin B-J, Yip AM, Hirsch GM. Frail patients are at increased risk for mortality and prolonged institutional care after cardiac surgery. Circulation. 2010;121:973-8.

33. Sündermann S, Dademasch A, Rastan A, Praetorius J, Rodriguez H, Walther T, et al. One-year follow-up of patients undergoing elective cardiac surgery assessed with the Comprehensive Assessment of Frailty test and its simplified form. Interact Cardiovasc Thorac Surg. 2011;13:119-23.

34. Sawatzky JA, Kehler DS, Ready AE, Lerner N, Boreskie S, Lamont D, et al. Prehabilitation program for elective coronary artery bypass graft surgery patients: a pilot randomized controlled study. Clin Rehabil. 2014;28:648-57.

35. Arthur HM, Daniels C, McKelvie R, Hirsh J, Rush B. Effect of a preoperative intervention on preoperative and postoperative outcomes in low-risk patients awaiting elective coronary artery bypass graft surgery. A randomized, controlled trial. Ann Intern Med. 2000;133:253-62.

36. Stammers AN, Kehler DS, Afilalo J, Avery LJ, Bagshaw SM, Grocott HP, et al. Protocol for the PREHAB study-Pre-operative Rehabilitation for reduction of Hospitalization After coronary Bypass and valvular surgery: a randomised controlled trial. BMJ Open. 2015;5:e007250.

37. Kumar K, Zarychanski R, Bell DD, Manji R, Zivot J, Menkis AH, et al. Impact of 24-hour in-house intensivists on a dedicated cardiac surgery intensive care unit. Ann Thorac Surg. 2009;88:1153-61.

38. Kumar K, Singal R, Manji RA, Zarychanski R, Bell DD, Freed DH, et al. The benefits of 24/7 in-house intensivist coverage for prolonged-stay cardiac surgery patients. J Thorac Cardiovasc Surg. 2014;148:290-7.e6.

Key Words: cardiac surgery, critical care, functional survival, octogenarian, rehospitalization

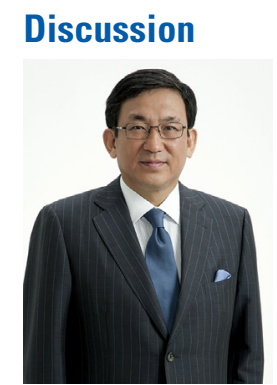

Dr K. Bando (Tokyo, Japan). This study is a natural extension of your previous study involving a cohort of 9545 adult patients that used a similar study design and was presented at the 2015 Society of Thoracic Surgeons meeting. You mentioned a 2-pronged approach to the improved outcomes for octogenarian patients in your article, preoperative referral, and management of the ICU period and transition to the community. However, I believe modifiable factors that can reduce the length of ICU stay and provide adequate postdischarge care are the most important considerations when looking to provide good late functional outcomes for these vulnerable patients.

My first question is related to preoperative patient characteristics. You mentioned that female gender and urgent and emergency procedures were more frequently seen in the prolonged ICU stay group. What about the Society of Thoracic Surgeons (STS) score or nutritional status defined by body mass index or other serum albumin level? Do you have any data indicating whether those factors may have any impact on the length of ICU stay or late functional survival?

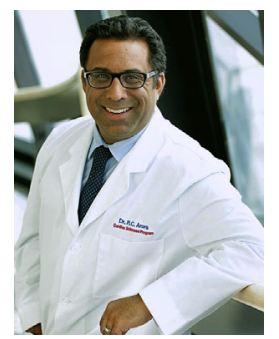

Dr R. C. Arora (Winnipeg, Manitoba, Canada). Although we collected European System for Cardiac Operative Risk Evaluation II for a portion of the study period, we didn't collect STS or STS Predicted Risk of Mortality on our patients, because this was not available for the entire duration of our patient cohort. With linkages to our ICU database, we are able to look at Acute Physiology And Chronic Health Evaluation II scores, which are shown for you in our Table 2. There are differences with the baseline Acute Physiology And Chronic Health Evaluation II scores.

Although Acute Physiology And Chronic Health Evaluation II is not specifically validated in a cardiac surgery patient population, it does provide some severity of illness information that may be more relevant to the ICU patient than specifically a preoperative cardiac surgery patient risk score.

Second, although a number of patient factors were considered, we acknowledge the limitation of not having detailed information on certain important contemporary issues (eg, frailty) or nutrition scores (eg, body mass index or albumin).

We are looking at ways to link this to laboratory data within our province, and we may have that ability in the future. The last several months, we've been collecting frailty data in a prospective fashion on elective patients going to the center, and we also hope to be able to present these data sometime in the future.

Dr Bando. As you know, the length of ICU stay was not determined only by preoperative factors. Did intraoperative factors, including pump time, crossclamp time, transfusion requirement, and surgeon's performance, have any impact on length of ICU stay and subsequent functional survival or readmission rate for those patients who are 80 years or older?

Dr Arora. A number of intraoperative factors (eg, transfusion pump time, crossclamp time) are considered, as shown for you in Table 2. However, they were considered as potential covariances in our model but were not significant. We don't specifically have a surgical performance 
measure or metric, but I'd be interested in learning more if you do something along those lines. But we don't specifically have a tool for that.

Dr Bando. When, how, and by whom is the decision to discharge those patients to their homes or other facilities made? Is it a team decision-making process consisting of members from different disciplines? Are there any standardized protocols for discharge? Is there any possibility of patients being discharged prematurely?

Dr Arora. I'll divide that into 2 groups: the ICU discharge and ward discharge, which likely play a role in both those factors. So as of 2007, we've had a different staffing model in which we have in-house consultant intensivists and cardiac anesthesiologist on a 24-hour basis that assess the patient as part of the morning interdisciplinary rounds. These rounds include the charge and bedside nurse, the respiratory therapist, physiotherapist, pharmacists, and so forth. We then will often reassess an individual patient several times during the course of the day to know if she or he meets (somewhat admittedly subjective) criterion for ICU discharge. Our actual return to ICU rates are relatively low. They're actually too small for us to examine because of the number of patients for this analysis.

For the ward to discharge home or to another institution, we've developed an interdisciplinary meeting that occurs twice a week to discuss discharge planning. That has been a process that has evolved in the last 3 to 4 years in which we involve the physician assistants, nurses, allied health team members, and home care to plan what services that may be required.

In general, I would suggest that we have a relatively conservative approach to discharge, because particularly in Manitoba, we serve a large geographic distance. To ensure that patients have the appropriate transportation and knowledge of who is receiving community-based practice are important considerations that may delay discharge. With regard to where patients are discharged, approximately $15 \%$ go to some other institution after our hospital, and it was more common in the patient with prolonged ICU LOS than those who had a typical course after surgery.

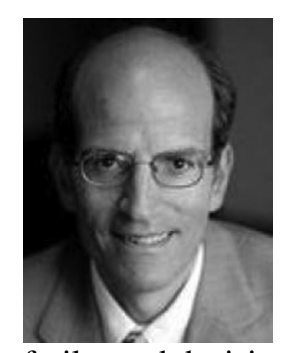

Dr G. Hirsch (Halifax, Nova Scotia, Canada). Congratulations on a welldone study and an important question as we all face increasing octogenarians with a lot of comorbidities and increasingly complex procedures. We've shown the same trends in Halifax, and as you know, we've been interested in frailty and decision making as you alluded to in this group. It's important to get at their ultimate fate as you're starting to get a handle on a difficult question to nail down because that's what an 80-year-old patient wants to know is am I going to get back to any functional capacity.

We both live in sort of centralized worlds of a single center serving an entire province. What mitigating strategies are you going to put in place for access to a physician who varies extremely between rural and almost no access back to the primary institution once they're discharged far away?

Dr Arora. Right. So this was part of the motivation for this question to start with in addition to changing in practice and now offering procedures to patients deemed previously inoperable with transcatheter aortic valve implantation programs and so forth that started after we did this analysis.

We looked at what cost the most as a way of trying to start to answer this problem, and a lot of that comes to their index hospital admission. When patients are discharged, although there is a high rate of return to hospital, the actual incremental cost of those are less. Therefore, it is reasonable to invest more effort or money spent up front and coordinate a short duration hospital stay and plan appropriately for hospital discharge.

We are currently in the process of developing prospectively a bidirectional communication tool with our community-based primary care practitioners to allow closing a loop with the information for discharge planning. Then when problems arise, we hope to have a clear process for reengaging the cardiac surgery center to answer questions or problems remotely or actually when to bring them back to the hospital so people don't slip through the cracks as time goes on. 


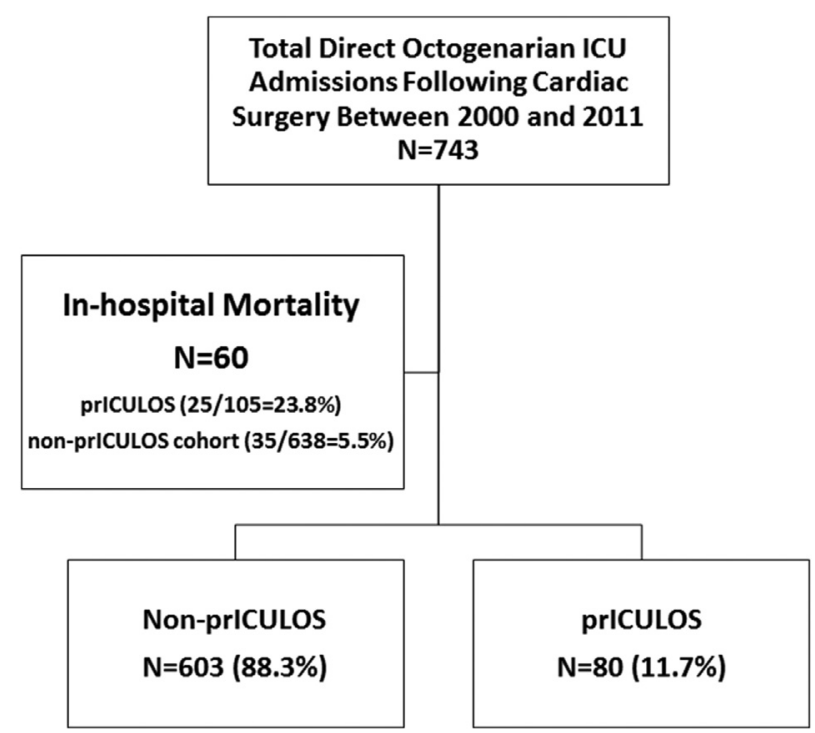

FIGURE E1. Patient cohort diagram. ICU, Intensive care unit; prICU$L O S$, prolonged intensive care unit length of stay. 
TABLE E1. Univariable Cox proportional hazards regression: Variables associated with functional survival time up to 1 year after discharge for octogenarians

\begin{tabular}{|c|c|c|c|}
\hline Variable & HR & $95 \% \mathrm{CI}$ & $P$ value \\
\hline \multicolumn{4}{|l|}{ Preoperative variables } \\
\hline Age & 1.03 & $0.93-1.15$ & .54 \\
\hline Female & 0.80 & $0.48-1.33$ & .39 \\
\hline BMI $\left(\mathrm{kg} / \mathrm{m}^{2}\right)$ & 1.01 & $0.95-1.07$ & .76 \\
\hline Previous MI & 0.83 & $0.51-1.36$ & .47 \\
\hline Diabetes mellitus & 2.17 & $1.31-3.61$ & $<.01$ \\
\hline Peripheral vascular disease & 1.38 & $0.80-2.37$ & .25 \\
\hline History of arrhythmia & 0.86 & $0.45-1.65$ & .66 \\
\hline Congestive heart failure & 0.90 & $0.47-1.73$ & .76 \\
\hline COPD & 1.25 & $0.60-2.63$ & .55 \\
\hline Renal insufficiency $(\mathrm{Cr}>1.8 \mathrm{mg} / \mathrm{dL})$ & 1.42 & $0.72-2.79$ & .31 \\
\hline Cerebrovascular disease & 1.24 & $0.65-2.36$ & .52 \\
\hline Previous mental health condition & 1.12 & $0.58-2.13$ & .74 \\
\hline Cardiogenic shock & 1.47 & $0.46-4.67$ & .52 \\
\hline \multicolumn{4}{|l|}{ Ejection fraction } \\
\hline $35 \%-49 \%$ vs $\geq 50 \%$ & 1.54 & $0.91-2.59$ & .11 \\
\hline$<35 \%$ vs $\geq 50 \%$ & 0.78 & $0.31-1.99$ & .60 \\
\hline Nonelective surgery & 2.85 & $1.49-5.46$ & $<.01$ \\
\hline Year of surgery & 0.96 & $0.90-1.03$ & .31 \\
\hline \multicolumn{4}{|l|}{ Operative variables } \\
\hline \multicolumn{4}{|l|}{ Procedure type } \\
\hline Single non-CABG vs isolated CABG & 1.32 & $0.70-2.51$ & .39 \\
\hline$\geq 2$ procedures vs isolated $\mathrm{CABG}$ & 1.36 & $0.77-2.39$ & .29 \\
\hline Crossclamp time (min) & 1.00 & $1.00-1.01$ & .48 \\
\hline Cardiopulmonary bypass time (min) & 1.00 & $1.00-1.01$ & .16 \\
\hline \multicolumn{4}{|l|}{ Postoperative variables } \\
\hline APACHE II $\geq 20$ & 2.32 & $1.39-3.87$ & $<.01$ \\
\hline Mechanical ventilation (d) & 1.20 & $1.09-1.32$ & $<.01$ \\
\hline Reintubation & 3.34 & $1.53-7.32$ & $<.01$ \\
\hline \multicolumn{4}{|l|}{ Blood products } \\
\hline Red blood cells (Units) & 1.12 & $1.06-1.18$ & $<.01$ \\
\hline Fresh-frozen plasma (Units) & 1.06 & $0.99-1.13$ & .09 \\
\hline Platelets (Units) & 1.08 & $1.04-1.13$ & $<.01$ \\
\hline \multicolumn{4}{|l|}{ ICU complications } \\
\hline Other $\mathrm{CV}$ procedure (sternal rewiring, returns to $\mathrm{OR}$, other) & 1.44 & $0.71-2.91$ & .31 \\
\hline Thrombocytopenia & 1.45 & $0.69-3.04$ & .32 \\
\hline Cardiogenic shock & 2.14 & $1.09-4.20$ & .03 \\
\hline Acute renal insufficiency $(\mathrm{Cr}>1.8 \mathrm{mg} / \mathrm{dL})$ & 2.98 & $1.42-6.24$ & $<.01$ \\
\hline \multicolumn{4}{|l|}{ LOS } \\
\hline ICU LOS (d) & 1.11 & $1.07-1.16$ & $<.01$ \\
\hline Prolonged ICU LOS ( $\geq 5 \mathrm{~d})$ & 2.35 & $1.32-4.19$ & $<.01$ \\
\hline Hospital LOS (d) & 1.02 & $1.02-1.03$ & $<.01$ \\
\hline \multicolumn{4}{|l|}{ Postoperative follow-up with any physician } \\
\hline No physician visits within $30 \mathrm{~d}$ of discharge vs $1-4$ visits & 4.98 & $2.90-8.56$ & $<.01$ \\
\hline $5+$ visits within $30 \mathrm{~d}$ of discharge vs $1-4$ visits & 1.23 & $0.55-2.75$ & .62 \\
\hline
\end{tabular}

$H R$, Hazard ratio; $C I$, confidence interval; $B M I$, body mass index; $M I$, myocardial infarction; $C O P D$, chronic obstructive pulmonary disease; $C r$, creatinine; $C A B G$, coronary artery bypass grafting; $A P A C H E$, Acute Physiology And Chronic Health Evaluation; $I C U$, intensive care unit; $C V$, cardiovascular; $O R$, operating room; $L O S$, length of stay. 